\title{
A Review of the Epidemiology of Breast Cancer in Asia: Focus on Risk Factors
}

\author{
Hyun Jo Youn ${ }^{1 *}$, Wonshik Han ${ }^{2}$
}

\begin{abstract}
Background and Aim: Breast cancer is the most prevalent cancer in women. To date, regional differences in breast cancer risk factors have not been identified. The aim of our review was to gain a better understanding of the role of risk factors in women with breast cancer in Asia. Methods: We conducted a PubMed search on 15 March 2016, for journal articles published in English between 2011 and 2016, which reported data for human subjects in Asia with a diagnosis of breast cancer. Search terms included breast neoplasm, epidemiology, Asia, prevalence, incidence, risk and cost of illness. Studies of any design were included, except for review articles and meta-analyses, which were excluded to avoid duplication of data. No exclusions were made based on breast cancer treatment. We reported the results using the Preferred Reporting Items for Systematic Reviews and Meta-analyses (PRISMA) guidelines. Results: A total of 776 abstracts were retrieved. After screening against the eligibility criteria, 562 abstracts were excluded. The remaining 214 abstracts, which were published between 2013 and 2015, were included in this review. Results were summarized and reported under three categories: incidence, prevalence or outcomes for breast cancer in Asia; modifiable risk factors; and non-modifiable risk factors. We found that the increased risk of breast cancer among participants from Asia was associated with older age, family history of breast cancer, early menarche, late menopause, high body mass index, being obese or overweight, exposure to tobacco smoke, and high dietary intake of fats or fatty foods. In contrast, intake of dietary fruits, vegetables, and plant- and soy-based products was associated with a decreased breast cancer risk. While based on limited data, when compared to women from the United States, women from Asia had a decreased risk of breast cancer. Conclusions: This review of 214 abstracts of studies in Asia, published between 2013 and 2015 , confirmed the relevance of known non-modifiable and modifiable risk factors for women with breast cancer.
\end{abstract}

Keywords: Breast neoplasms- epidemiology- Asia- risk factors

Asian Pac J Cancer Prev, 21 (4), 867-880

\section{Introduction}

The most recent data from the World Health Organization (WHO) shows that breast cancer (BC) is the most prevalent cancer in women, with an incidence of 1.7 million cases in 2012 ( $25 \%$ of all female cancers). It is the fifth leading cause of death from cancer overall (International Agency for Research on Cancer, 2016). The incidence is similar in regions identified by the WHO as more- and less-developed, at 13\% $(788,000)$ and $11 \%$ $(883,000)$ respectively (International Agency for Research on Cancer, 2016). WHO figures from 2012 estimate the international 5-year prevalence for BC at 6,232,000; by region (as defined by WHO) the rate in South-East Asia is 735,000 compared to 1,936,000 in Europe and 1,618,000 in the Americas (International Agency for Research on Cancer, 2016).

Identified risk factors for BC may be non-modifiable such as sex, age, genetic characteristics including family or personal history of $\mathrm{BC}$, ethnicity, and early menarche or menopause. Modifiable risk factors, usually associated with lifestyle factors, can include alcohol consumption, excess weight or obesity, physical inactivity, parity, and use of some medications, such as oral contraceptives (American Cancer Society, 2016; World Health Organization, 2016).

Despite these associations, women with risk factors for $\mathrm{BC}$ may never go on to develop $\mathrm{BC}$ and many women with $\mathrm{BC}$ have no known risk factors. Therefore, it is difficult to definitively determine the contribution of individual risk factors to the development of BC (American Cancer Society, 2016).

To date, regional differences in $\mathrm{BC}$ risk factors have not been identified. The aim of our review was to gain a better understanding of the role of risk factors in women with $\mathrm{BC}$ in Asia.

${ }^{I}$ Department of Surgery, Research Institute of Clinical Medicine, Chonbuk National University and Biomedical Research Institute, Chonbuk National University Hospital, ${ }^{2}$ Department of Surgery and Cancer Research Institute, Seoul National University College of Medicine, Seoul National University Cancer Hospital, Republic of Korea. *For Correspondence: yhj0903@jbnu.ac.kr 


\section{Materials and Methods}

We performed a systematic review of the current body of evidence available through PubMed. The search was conducted on 15 March 2016. Medical subject headings (MeSH) search terms used included: breast neoplasm, epidemiology, Asia, prevalence, incidence, risk and cost of illness. The search was limited to human studies, published in English between 2011 and 2016. Studies were included in our review if the participants had BC of any age, histopathology, grade or stage, and received treatment of any type; studies needed to report information on epidemiology, prevalence, incidence, risk factors, and cost of illness. Our review included all study designs, except for review articles and meta-analyses, which were excluded to avoid duplication of data. Studies were only included if they reported data from countries within South East Asia, Far East Asia, or Western Asia excluding the Middle East. Articles were excluded if their primary objective was to examine the correlation of $\mathrm{BC}$ to other types of cancer or to examine surgical treatments for BC.

\section{Results}

Our search identified 783 articles; seven articles were excluded due to no abstract being available. We did not find any articles published in 2016. To reduce the volume of data identified in our search, only articles published within the previous three years (2013-2015) were included. This resulted in 169 eligible articles being excluded based on publication date (2011 and 2012). After the exclusion of duplicates and articles that did not meet the inclusion criteria, a total of 214 remaining articles were included in this review. The flow diagram of the literature selection process is presented in Figure 1.

Results have been summarized and reported under three categories: incidence, prevalence or outcomes for breast cancer in Asia; modifiable risk factors; and non-modifiable risk factors. A summary of studies showing risk and protective factors influencing changes in BC risk is provided in Supplementary Table 2; a visual representation of risk and protective factors is provided in Figure 2 and Figure 3. Due to the large number of studies eligible for review, descriptive data without an association with altered $\mathrm{BC}$ risk have not been provided in the tables. Key trends are summarized in the sections below.

\section{Incidence, prevalence or outcomes for breast cancer in Asia}

$\mathrm{BC}$ was reported as the leading type of cancer in females in Japan (Matsuda et al., 2014; Hori et al., 2015), and China (Zhou et al., 2015). In addition, the incidence of $\mathrm{BC}$ was shown to increase over time in China (Wu et al., 2014b; Zhou et al., 2015), India (Asthana et al., 2014) and Thailand (Virani et al., 2014), although it was unclear if this was due to improved identification (i.e. screening and detection) or a true reflection of increased incidence. While BC incidence is predicted to increase in Hong Kong by 2025 , mortality is projected to decrease from 9.3 to 8.6 per 100,000 women, respectively (Wong et al., 2015). This may be related to Hong Kong's socio-economic development, and the improvements in survival may be due to treatment advancement and improved health service delivery (Wong et al., 2015). This is in contrast to China, where BC mortality increased over a 5-year period (annual percentage change of $6.89 \%$ ) between 2006 and 2010 (Wu et al., 2014b). In one study from Malaysia, almost all $(n=1453,99.2 \%)$ had an estimated 10-year risk of BC of less than 2\% (Hassan et al., 2015). In Korea, the rate of 5-year recurrence-free survival (RFS) was 90.8\%, 10-year RFS was $81.9 \%$, and 5-year overall survival (OS) was 94.6\% (Kim et al., 2014; Park et al., 2015b). However other studies from Korea have reported the 10-year OS rate at $86.9 \%$ (Park et al., 2015b) and another at $61.4 \%$ (Jung et al., 2013). In Hong Kong and Vietnam, 5-year OS rates were $90.5 \%$ and $74 \%$, respectively (Lan et al., 2013; Kwong et al., 2014). The 5-year OS rate was similar in male BC patients from Hong Kong $(n=132,78.7 \%)$ (Kwong et al., 2014). Supplementary Figure 1 Flow diagram of the literature selection process

\section{Dietary Factors}

Dietary factors associated with increased BC risk included intake of total n-6 polyunsaturated fatty acids (PUFA) (Kiyabu et al., 2015), vitamin D deficiency or insufficiency (Shi et al., 2014a; Park et al., 2015a), high levels of serum cadmium (Nagata et al., 2013; Itoh et al., 2014; Ding et al., 2015), high intake of salt (Park et al., 2014b), sugar (Sulaiman et al., 2014), meat (Ko et al., 2013), and saturated fat and oils (Balasubramaniam et al., 2013; Wang et al., 2013a). In women with BC, the trace elements, cadmium, magnesium, copper, cobalt and lithium, were found in high levels. This suggests a possible association between serum levels of trace elements and breast cancer risk, however, this requires further investigation and if confirmed, the modulation of trace elements may help to reduce breast cancer risk (Ding et al., 2015).

High intake of dietary fiber was associated with decreased BC risk (Sulaiman et al., 2014) along with high levels of $\alpha$-carotene, $\beta$-carotene, $\beta$-cryptoxanthin, lutein/ zeaxanthin (Wang et al., 2014a), and soy products or isoflavones (Ko et al., 2013; Li et al., 2013a; Wada et al., 2014) (Table 2). In addition, BC patients had significantly lower levels of manganese, aluminum, iron and titanium than women who did not have BC (Ding et al., 2015). High consumption of fruit and vegetables has also been linked to a reduction in breast cancer risk in Asia. (Sangrajrang et al., 2013)

In one study, the consumption of crucian carp was associated with increased BC risk, but total freshwater fish intake and consumption of black carp and silver carp were associated with decreased risk (Gao et al., 2014).

\section{Location of residence}

Location of residence were identified as a modifiable factor associated with increased BC risk and was identified in three papers (Shi et al., 2014b; Wu et al., 2014b; Fei et al., 2015). Results of the studies identified that living in an urban region of China (versus a rural region) led to an increased $\mathrm{BC}$ risk. One paper noted a higher incidence of $\mathrm{BC}$ was correlated to higher population density, percentage 


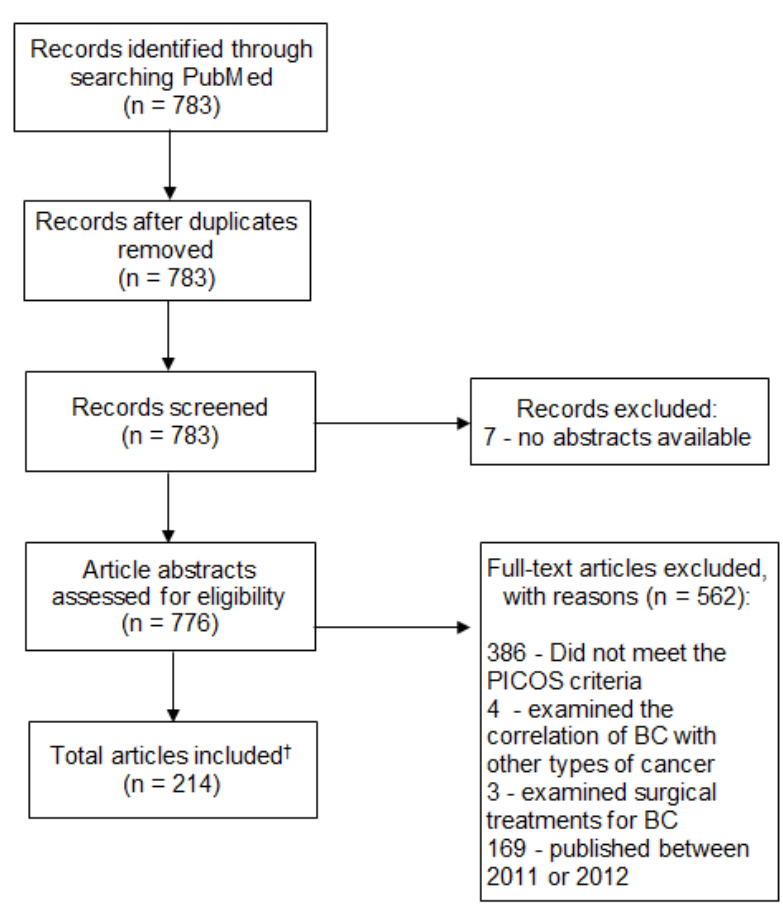

Figure 1. Flow Diagram of the Literature Selection Process. NA, not applicable; * No other sources were searched; $\dagger$, Due to the volume of data available for analysis, results were analyzed based only on the content of study abstracts; BC, breast cancer

of non-agricultural population, and second industry output; in contrast, incidence was negatively correlated with the percentage of population employed in primary industry (Fei et al., 2015). Other modifiable risk factors including active or passive smoking (Mizoo et al., 2013; Pimhanam et al., 2014; Tong et al., 2014; Wada et al., 2015), high body mass index (BMI) (Mizoo et al., 2013; Sangrajrang et al., 2013; Suzuki et al., 2013; Wang et al., 2013b; Anothaisintawee et al., 2014; Wada et al., 2014; Fu et al., 2015), use of oral contraceptives (Bhadoria et al., 2013; Anothaisintawee et al., 2014; Poosari et al., 2014), high perceived level of stress, and low level of physical

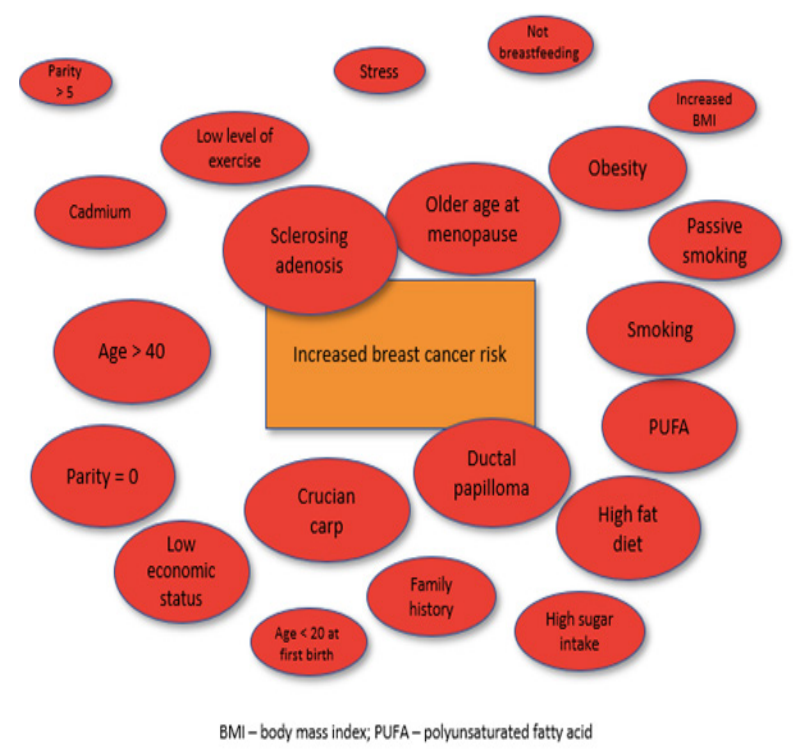

Figure 2. Risk Factors for Breast Cancer in Asia activity (Wang et al., 2013a) were discussed in the papers.

\section{BMI and physical activity}

A Thai study evaluated the relationship between BC risk and obesity and physical activity. The study found that being underweight at ages 10 and 20 years showed a reduced risk of $\mathrm{BC}$ in all women $(\mathrm{OR}=0.70,95 \% \mathrm{CI}$ $0.56-0.88$ and $\mathrm{OR}=0.74,95 \% \mathrm{CI} 0.59-0.93$, respectively) and undertaking regular physical activity also showed a reduced risk $(\mathrm{OR}=0.78,95 \%$ CI 0.68-0.98). When reviewed by type of physical activity undertaken, those who had the highest levels of walking had the greatest reduction in risk $(\mathrm{OR}=0.58,95 \%$ CI $0.38-0.88)$ (Sangrajrang et al., 2013).

\section{Reproductive history and breastfeeding}

Several of the identified studies have investigated the effect of parity, abortion, age at pregnancy and history of breastfeeding on BC risk. In a Korean study, parity of five or more increased the risk of luminal $\mathrm{A}$ and $\mathrm{B}$ breast cancer (HR 1.95, 95\% CI, 0.96-3.97, $\mathrm{p}=0.0055$; and HR 1.12; $95 \%$ CI, 0.42-3.02, $\mathrm{p}=0.0073$ respectively) and an early age of first birth (less than 20 years) was associated with increased risk of luminal A BC recurrence (HR 1.61, 95\% CI, 0.62-4.26; $p=0.039$ ). However, between one and three childbirths was associated with decreased risk of luminal A and $\mathrm{B}$ (HR, 0.56; 95\% CI, 0.34-0.91, $\mathrm{p}=0.0055$; and HR, $0.32 ; 95 \%$ CI, 0.17-0.61, $\mathrm{p}=0.0073$ respectively) (Lee and Oh, 2014). In India, women with BC, had a higher age at last child birth, lower mean duration of breastfeeding, and a higher number of abortions compared to women who did not have BC (Bhadoria et al., 2013). In China, Wu et al., (2014a) reported reduced BC risk in parous women with 3 or more induced abortions or 3 or more induced abortions after first live birth, compared with parous women without induced abortion. Also in China, age over 25 years at first full-term pregnancy was associated with increased risk of $\mathrm{BC}$, but a history of full-term pregnancy was associated with a decreased risk of BC (Pei et al., 2014). Huang et al., (2014) reported that among participants from China,

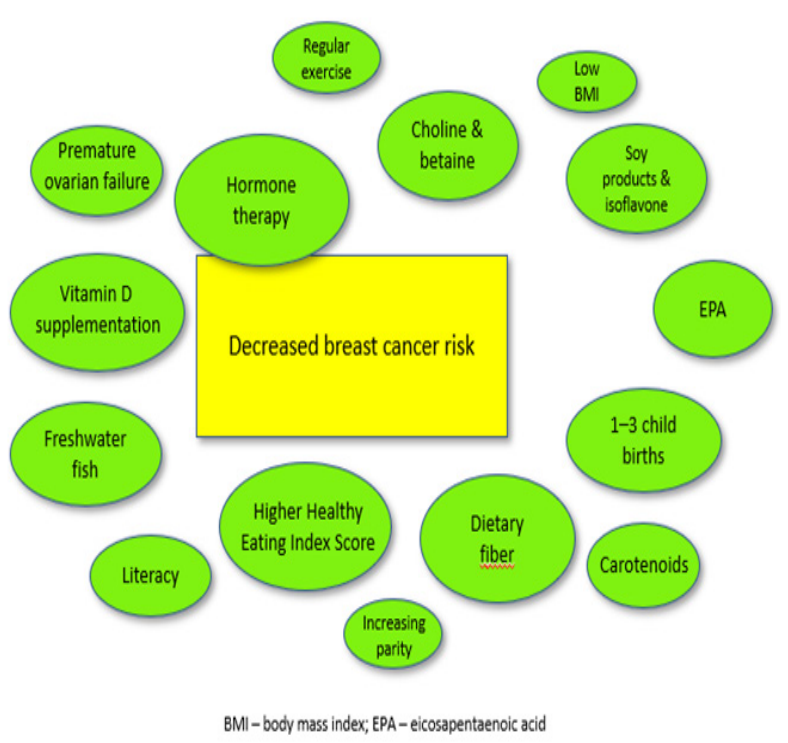

Figure 3. Protective Factors for Breast Cancer in Asia 
later age at either first live birth, or at first pregnancy and last pregnancy were associated with increased BC risk ( $\mathrm{p}$-trend $=0.002,0.015,0.008$, respectively). Among participants from Japan, BC was increased in women who performed mixed feeding (HR 1.12, 95\% CI, 0.92-1.37, $\mathrm{p}$-trend $=0.014$ ) or feeding only with formula (HR 1.80 , 95\% CI, 1.14-2.86, p-trend $=0.014$ ) compared with women who only breastfed (Sugawara et al., 2013).

\section{Breast cancer risk by geographic location}

Several studies demonstrated differences in the prevalence and mortality associated with $\mathrm{BC}$ between nations in the Asian region, and significantly, differences within individual nations. Ginsburg et al., (2017) argue that the majority of women who die from $\mathrm{BC}$ live in low-income countries; this is in contrast to high-income countries where most women who are diagnosed with BC will survive. Malvia et al., (2017) indicate that the overall age adjusted rates for $\mathrm{BC}$ in India is as high as 25.8 per 100,000 women but lower than that of the United Kingdom (95 per 100,000). Indian mortality rates were reported at 12.7 per 100,000 women, which is comparable to the $\mathrm{BC}$ mortality rates in the UK (17.1 per 100,000). Further, disparities in BC risk and mortality can be seen within individual countries in Asia and within different regions within the same country. When Indian $\mathrm{BC}$ mortality rates are reported by region, some regions reported rates as high as 41 per 100,000 in Delhi and as low as 12.4 per 100,000 in Barshi rural region (Malvia et al., 2017).

Non-modifiable risk factors for breast cancer in Asia Age In India, the mean age of $\mathrm{BC}$ diagnosis was 53 years (Tulsyan et al., 2014), and the median age was 45 years (Krishnatreya et al., 2014). This was similar to data from China, which indicated that the estimated risk of recurrent breast cancer increased with age (mean $=46$ years) (Chen et al., 2014b). Peaks in age-specific breast cancer incidence were at 50-59 years and $>85$ years (Wu et al., 2014b). In a Thai study, incidence was projected to increase at a higher rate in women aged 50 years or over compared to those aged under 50 years (period to 2029) (Virani et al., 2014). Other studies have reported that benign breast disease was more likely to occur in women aged under 40 years, with BC more likely in women aged over 40 years (Kotepui and Chupeerach, 2013; Kotepui et al., 2014).

\section{Family history}

In China (Zhou et al., 2013; Chen et al., 2014b; Lee et al., 2014; Pei et al., 2014), Pakistan (Shamsi et al., 2013), Hong Kong (Tse et al., 2015), India (Bhadoria et al., 2013), and Korea (Park et al., 2014a), a family history of $\mathrm{BC}$ was associated with an increased risk of BC. In Hong Kong, risk of BC (in women with estrogen receptor-positive tumors) was higher in women with an affected mother $(\mathrm{OR}=3.97,95 \% \mathrm{CI}: 1.46-10.79)$ when compared to women with an affected sister $(\mathrm{OR}=2.06$, 95\%CI: 1.07-3.97) (Tse et al., 2015). A study in Nepal showed that family history of $\mathrm{BC}$ was evident in a higher proportion of women who developed $\mathrm{BC}$ aged less than 40 years (3\%) compared with those aged 40 years or over
(0.3\%) (Thapa et al., 2013).

\section{Ethnicity}

Race/ethnicity and age-specific incidence rates have been shown to increase continuously until 80 years in white women from the United States (US), but plateau or decrease after 50 years in Asian women (Sung et al., 2015). A greater absolute risk for all BC subtypes has been found in women from the US National Cancer Institute's Surveillance, Epidemiology, and End Results 18 registries database (SEER 18) compared to those from Malaysia (Horne et al., 2015). A further study demonstrated that, following adjustment for population structure, the median age of BC diagnosis was lower in China (50-54 years) than in the United States and European Union (55-59 years) (Song et al., 2014b). The authors suggest that this is due to racial differences in genetics and lifestyle and that BC screening programs should be commenced at an earlier age for Chinese women (Song et al., 2014b).

\section{Reproductive health}

Menopausal status was associated with $\mathrm{BC}$ risk in studies conducted in Thailand (Anothaisintawee et al., 2014), India (Chattopadhyay et al., 2014), Pakistan (Sohail et al., 2013), and Korea (Park et al., 2013). Among participants from India, compared with controls, women with $\mathrm{BC}$ had a lower mean age at menarche $(13.20 \pm 1.33$ and $14.58 \pm 0.85$ respectively, $\mathrm{p}=0.00)$ and a later age of menopause $(49.38 \pm 5.21$ and $47.89 \pm 3.96$ respectively, $\mathrm{p}=0.002$ ) (Bhadoria et al., 2013). Lee et al., (2014) reported that late menopause was significantly associated with increased BC risk. In Korea, age at menarche and menopausal status or age at menopause were risk factors for BC (Park et al., 2013). A second study in Korea confirmed the association with early age at menarche and BC risk, irrespective of tumor subtype (Chung et al., 2013). In Pakistan, older age at menopause was associated with increased BC risk (Shamsi et al., 2013).

\section{Hormone receptor status}

In China, estrogen levels and estrogen receptor-mediated serum bioactivity were higher in BC cases than controls (Lim et al., 2014). A study conducted in Malaysia found that the majority of BC cases were hormone receptor-negative (Horne et al., 2015). Conversely in a Korean study, $69.5 \%$ of BCs were hormone receptor-positive (Song et al., 2014a) and $44.6 \%$ of BCs were estrogen receptor-positive in an Indian study (Singh et al., 2014). Among participants from Korea, when estrogen receptor-positive/progesterone receptor-positive tumors were compared to estrogen receptor-positive/progesterone receptor-negative tumors, women with the latter were 4 years older at diagnosis and more likely to be postmenopausal (Chung et al., 2013).

\section{Genetic risk factors}

Several of the studies investigated the association between gene polymorphisms and $\mathrm{BC}$ risk. In addition, there were also studies investigating the association between BC risk and DNA methylation (Kuchiba et al., 2014), length of CAG repeats (of androgen receptor 
Table 1. Summary of Incidence, Prevalence or Outcomes Data for Breast Cancer in Asia

\begin{tabular}{|c|c|c|}
\hline Country & References & Reported data \\
\hline China & $\begin{array}{l}\text { Zhou HB, et al.(Zhou et al., 2015); } \\
\text { Wu LZ, et al.(Wu et al., 2014b); Shi } \\
\text { XJ, et al.(Shi et al., 2014b); Zhang X, } \\
\text { et al.(Zhang et al., 2013b); Li XP, et } \\
\text { al.(Li et al., 2013b) }\end{array}$ & $\begin{array}{l}\text { - Crude incidence rate of } 20.0 / 100,000 \text { population between } 2007 \text { and } 2012 \text {. } \\
\text { - The age-standardized rate was } 21.1 / 100,000 \text { in } 2012 \text {. } \\
\text { - Average annual percentage change of } 11.3 \% \text {. } \\
\text { - Mortality rate has increased yearly since } 1991 \text {, a trend predicted to con- } \\
\text { tinue over the following } 5 \text { years. } \\
\text { - After median } 60 \text { months of follow-up (range, } 8-60 \text { months), local and } \\
\text { distant recurrence of breast cancer was observed in } 23.4 \% \text { of cases. } \\
\text { - Between } 2002 \text { and } 2006 \text {, the } 5 \text {-year relative survival rate for breast cancer } \\
\text { was }>40 \% \text {. } \\
\text { In Jiangsu between } 2006 \text { and } 2010 \text { : } \\
\text { - } 11,013 \text { new cases of female breast cancer and } 3,068 \text { deaths due to female } \\
\text { breast cancer were identified. } \\
\text { - The annual average crude incidence and age-standardized incidence were } \\
25.2 \text { and } 17.9 \text { per } 100,000 \text {, and mortality rates were } 7.03 \text { and } 4.81 \text { per } \\
100,000 \text { respectively. } \\
\text { - Incidence and mortality increased with annual percentage changes of } \\
4.47 \% \text { and } 6.89 \% \text {, respectively. }\end{array}$ \\
\hline Japan & $\begin{array}{l}\text { Hori M, et al.(Hori et al., 2015); Suga- } \\
\text { wara Y, et al.(Sugawara et al., 2013); } \\
\text { Kasahara Y, et al.(Kasahara et al., } \\
\text { 2013); Yamashiro H, et al.(Yamashiro } \\
\text { et al., 2014); Matsuda A, et.al.(Mat- } \\
\text { suda et al., 2014); Wada K, et.al.(Wada } \\
\text { et al., 2014) }\end{array}$ & $\begin{array}{l}\text { - Leading type of cancer in females. } \\
\text { - Primary invasive cancer incidence } 775,601 \text { in } 2009 \text {. } \\
\text { - Of } 26,680 \text { women, } 148 \text { incident cases of breast cancer were identified dur- } \\
\text { ing } 11 \text { years of follow-up. } \\
\text { - The rate of breast cancer detected in women aged } 40-49 \text { years was } 0.28 \% \text {. } \\
\text { - Bone metastases developed in } 11.3 \% \text { ( }=193 \text { ) of recently diagnosed breast } \\
\text { cancer cases } \\
\text { - The bone-metastasis-free rate at } 5 \text { years was } 89.2 \% \text {. }\end{array}$ \\
\hline $\begin{array}{l}\text { Hong } \\
\text { Kong }\end{array}$ & $\begin{array}{l}\text { Kwong A, et al.(Kwong et al., 2014); } \\
\text { Wong IO, et al.(Wong et al., 2015) }\end{array}$ & $\begin{array}{l}\text { - Mean age at diagnosis of breast cancer in male and female patients was } \\
64.5 \text { and } 52.7 \text { years, respectively. } \\
\text { - The 5-year OS, breast-cancer-specific mortality, and DFS for male and } \\
\text { female patients were } 78.7 \%, 90.5 \%, 90.5 \% \text {, and } 77.9 \%, 86.4 \% \text {, and } 81.4 \% \\
\text { respectively. } \\
\text { - Age-standardized incidence projected to increase from } 56.7 \text { per } 100,000 \\
\text { women in } 2011-2015 \text { to } 62.5 \text { per } 100,000 \text { women in } 2021-2025 \text {. } \\
\text { - Age-standardized mortality projected to decrease from } 9.3 \text { to } 8.6 \text { per } \\
100,000 \text { women for the same periods. }\end{array}$ \\
\hline Korea & $\begin{array}{l}\text { Park YH, et al.(Park et al., 2015b); } \\
\text { Kim JH, et al.(Kim et al., 2014); } \\
\text { Jung M, et al.(Jung et al., 2013) }\end{array}$ & $\begin{array}{l}\text { - During a median follow-up of } 100 \text { months, the } 5 \text {-year RFS rate and OS } \\
\text { rate were } 90.8 \% \text { and } 94.6 \% \text {, respectively. } \\
\text { - The } 10 \text {-year estimated RFS rate and OS rate were } 81.9 \% \text { and } 86.9 \% \text {, } \\
\text { respectively. } \\
\text { - After median follow-up of } 82 \text { months, } 5 \text {-year local RFS was } 98.4 \% \text { and the } \\
10 \text {-year local RFS was } 95.8 \% \text { for all patients. Nineteen patients }(2.6 \%) \text { had } \\
\text { ipsilateral breast recurrences, including } 12 \text { invasive recurrences and seven } \\
\text { DCIS. Nine patients ( } 1.2 \% \text { ) patients developed contralateral breast cancer, } \\
\text { including six invasive breast cancer and three DCIS. } \\
\text { - The observed } 10 \text {-year OS, breast cancer-specific survival and event-free } \\
\text { survival was } 61.4 \%, 62.3 \% \text { and } 59.1 \% \text {, respectively. }\end{array}$ \\
\hline Thailand & $\begin{array}{l}\text { Virani S, et al.(Virani et al., 2014); } \\
\text { Kotepui M, et.al.(Kotepui et al., 2014) }\end{array}$ & $\begin{array}{l}\text { - Age-adjusted incidence rate increased by almost } 300 \% \text { from } 1990 \text { to } 2010 \text {. } \\
\text { - Incidence rates increased from } 10.0 \text { to } 27.8 \text { cases per } 100,000 \text { person- } \\
\text { years. } \\
\text { - Benign breast disease and fibroadenoma occurred in women aged }<40 \\
\text { years whereas breast cancer occurred in women aged }>40 \text { years (OR: } 8.629 \text {; } \\
95 \% \text { CI: } 6.939-10.729 ; \mathrm{P}<0.001 \text { and OR: } 23.906 ; 95 \% \text { CI: } 18.359-31.129 \text {; } \\
\text { P }<0.001 \text {, respectively). } \\
\text { - Fibrocystic change occurred in patients aged }<40 \text { years whereas breast } \\
\text { cancer commonly occurred in patients aged } \geq 40 \text { years (OR: } 3.865 ; 95 \% \text { CI: } \\
2.993-4.991 ; P<0.001 \text { ). }\end{array}$ \\
\hline India & Asthana S, et al.(Asthana et al., 2014) & $\begin{array}{l}\text { - The annual percentage change in incidence for breast cancer ranged from } \\
0.46 \% \text { to } 2.56 \% \text {. }\end{array}$ \\
\hline Asia & $\begin{array}{l}\text { Keramatinia A, et al.(Keramatinia et } \\
\text { al., 2014) }\end{array}$ & $\begin{array}{l}-23,661 \text { cases of breast cancer occurred in the } 10 \text { analyzed registries over } \\
32 \text { years of follow-up }(1970-2002) \text {. }\end{array}$ \\
\hline Malaysia & Hassan N, et al.(Hassan et al., 2015) & $\begin{array}{l}\text { - Of the Chinese women in this study, } 99.2 \% \text { had a } 10 \text {-year risk of breast } \\
\text { cancer of }<2 \% \text {. }\end{array}$ \\
\hline Vietnam & Lan NH, et al.(Lan et al., 2013) & $\begin{array}{l}\text { - OS rates at } 1,3 \text {, and } 5 \text { years following diagnosis were } 0.94,0.83 \text { and } 0.74 \\
\text { respectively. }\end{array}$ \\
\hline
\end{tabular}


Table 2. Summary of Studies Reporting Risk Factors and Protective Factors for Breast Cancer in Asia

\begin{tabular}{|c|c|c|c|c|}
\hline Factor & Studies & Comparison & $\begin{array}{l}\text { Reported risk (odds ratio, relative } \\
\text { risk or hazard ratio [95\% Confidence } \\
\text { interval]) }\end{array}$ & $\begin{array}{l}\text { Effect } \\
\text { measure }\end{array}$ \\
\hline & & RISK FACTORS & & \\
\hline \multicolumn{5}{|l|}{ Non-modifiable } \\
\hline Sclerosing adenosis & $\begin{array}{l}\text { Chen JJ, et al.(Chen et al., } \\
\text { 2014b) }\end{array}$ & $\begin{array}{l}\text { No comparator - retrospective } \\
\text { file review of operable bilateral } \\
\text { breast cancer cases. }\end{array}$ & $11.8(5.3,26.3)$ & HR \\
\hline $\begin{array}{l}\text { Inflammatory bowel } \\
\text { disease requiring } \\
\text { hospitalization more than } \\
\text { twice per year }\end{array}$ & Tsai MS, et al.(Tsai et al., 2015) & $\begin{array}{l}\text { No inflammatory bowel disease } \\
\text { symptoms vs inflammatory bowel } \\
\text { disease symptoms }\end{array}$ & $8.45(4.64,15.4)$ & HR \\
\hline Ductal papilloma & Pan L, et al.(Pan et al., 2013) & $\begin{array}{l}\text { Normal breast ultrasound vs } \\
\text { abnormal breast }\end{array}$ & $6.52(1.87,22.75)$ & OR \\
\hline Older age at menopause & $\begin{array}{l}\text { Shamsi U, et al.(Shamsi et al., } \\
\text { 2013) }\end{array}$ & Breast cancer vs no breast cancer & $\begin{array}{l}\text { Age }<45 \text { years } 3.92(2.52,6.18) \\
\text { Age }>45 \text { years } 6.42(3.47,11.98)\end{array}$ & OR \\
\hline Age $>40$ years & $\begin{array}{l}\text { Kotepui M, et al.(Kotepui and } \\
\text { Chupeerach, 2013) }\end{array}$ & $\begin{array}{l}\text { No comparator - retrospective } \\
\text { file review of all breast cancer } \\
\text { cases. }\end{array}$ & $3.87(2.99,4.99)$ & OR \\
\hline $\begin{array}{l}\text { Lobular carcinoma } \\
\text { component involvement }\end{array}$ & $\begin{array}{l}\text { Chen JJ, et al.(Chen et al., } \\
\text { 2014b) }\end{array}$ & $\begin{array}{l}\text { No comparator - retrospective } \\
\text { file review of operable bilateral } \\
\text { breast cancer cases. }\end{array}$ & $5.6(2.6,12.1)$ & HR \\
\hline Diabetes & $\begin{array}{l}\text { Chen YK, et al.(Chen et al., } \\
\text { 2013b) }\end{array}$ & $\begin{array}{l}\text { Graves' disease vs no Graves' } \\
\text { disease }\end{array}$ & $3.35(1.02,11.01)$ & OR \\
\hline Multiple sclerosis & $\begin{array}{l}\text { Sun LM, et al.(Sun et al., } \\
\text { 2014c) }\end{array}$ & $\begin{array}{l}\text { Multiple sclerosis vs no Multiple } \\
\text { sclerosis }\end{array}$ & $2.23(1.11,4.46)$ & HR \\
\hline $\begin{array}{l}\text { Family history of breast } \\
\text { cancer }\end{array}$ & $\begin{array}{l}\text { Zhou W, et al.(Zhou et al., } \\
\text { 2013) } \\
\text { Chen JJ, et al.(Chen et al., } \\
\text { 2014b) }\end{array}$ & $\begin{array}{l}\text { No comparator - retrospective } \\
\text { file review of operable bilateral } \\
\text { breast cancer cases. }\end{array}$ & $\begin{array}{l}2.11(1.26,3.52) \\
\text { First-degree relatives } 2.0(1.1,3.4)\end{array}$ & $\begin{array}{l}\text { OR } \\
\text { HR }\end{array}$ \\
\hline $\begin{array}{l}\text { Polycystic ovary } \\
\text { syndrome }\end{array}$ & $\begin{array}{l}\text { Shen CC, et al.(Shen et al., } \\
\text { 2015) }\end{array}$ & $\begin{array}{l}\text { Polycystic ovary syndrome vs no } \\
\text { Polycystic ovary syndrome }\end{array}$ & $1.99(1.05,3.77)$ & HR \\
\hline \multicolumn{5}{|l|}{ Modifiable } \\
\hline \multicolumn{5}{|l|}{ Demographics } \\
\hline Low economic status & Kim SH, et al.(Kim et al., 2013) & $\begin{array}{l}\text { No comparator - descriptive } \\
\text { study }\end{array}$ & 2.22 (not reported) & OR \\
\hline Single marital status & $\begin{array}{l}\text { Shamsi U, et al.(Shamsi et al., } \\
\text { 2013) }\end{array}$ & Breast cancer vs no breast cancer & $1.55(1.10,2.39)$ & OR \\
\hline Married & Lan NH, et al.(Lan et al., 2013) & $\begin{array}{l}\text { No comparator - retrospective } \\
\text { data review }\end{array}$ & $1.59(1.09,2.33)$ & HR \\
\hline $\begin{array}{l}\text { Age }<20 \text { years at first } \\
\text { birth }\end{array}$ & $\begin{array}{l}\text { Lee JS, et al.(Lee and Oh, } \\
\text { 2014) }\end{array}$ & $\begin{array}{l}\text { No comparator - retrospective } \\
\text { data review }\end{array}$ & Luminal A $1.61(0.62,4.26)$ & HR \\
\hline Parity $>5$ & $\begin{array}{l}\text { Lee JS, et al.(Lee and Oh, } \\
\text { 2014) }\end{array}$ & $\begin{array}{l}\text { No comparator - retrospective } \\
\text { data review }\end{array}$ & $\begin{array}{l}\text { Luminal A } 1.95(0.96,3.97) \\
\text { Luminal B } 1.12(0.42,3.02)\end{array}$ & HR \\
\hline Not breastfeeding & $\begin{array}{l}\text { Sugawara Y, et al.(Sugawara et } \\
\text { al., 2013) }\end{array}$ & Mixed feeding vs formula feeding & $\begin{array}{l}\text { Mixed feeding } 1.12(0.92,1.37) \\
\text { Formula only } 1.80(1.14,2.86)\end{array}$ & HR \\
\hline \multicolumn{5}{|l|}{ Lifestyle } \\
\hline Past or current smoker & Kim SH, et al.(Kim et al., 2013) & $\begin{array}{l}\text { No comparator - descriptive } \\
\text { study }\end{array}$ & 3.77 (not reported) & OR \\
\hline Obesity & $\begin{array}{l}\text { Noh HM, et al.(Noh et al., } \\
\text { 2013) }\end{array}$ & Breast cancer vs no breast cancer & Postmenopausal $2.24(1.22,4.10)$ & OR \\
\hline $\begin{array}{l}\text { Less than } 1,000 \mathrm{kcal} \\
\text { of physical activity } \\
\text { expenditure per week }\end{array}$ & $\begin{array}{l}\text { Wang L, et al.(Wang et al., } \\
\text { 2013a) }\end{array}$ & Breast cancer vs no breast cancer & $2.17(1.39,3.39)$ & OR \\
\hline Nulliparity & $\begin{array}{l}\text { Balasubramaniam SM, et } \\
\text { al.(Balasubramaniam et al., } \\
\text { 2013) }\end{array}$ & Breast cancer vs no breast cancer & $2.4(1.14,5.08)$ & OR \\
\hline Insulin use $\geq 3$ years & Tseng CH.(Tseng, 2015) & $\begin{array}{l}\text { No comparator- data from } \\
\text { National Health Insurance } \\
\text { Program. }\end{array}$ & $2.01(1.1,3.65)$ & HR \\
\hline \multirow[t]{3}{*}{ Passive smoking } & $\begin{array}{l}\text { Pimhanam C, et al.(Pimhanam } \\
\text { et al., 2014) }\end{array}$ & Breast cancer vs no breast cancer & $3.77(1.11 .12 .82)$ & OR \\
\hline & $\begin{array}{l}\text { Tong JH, et al.(Tong et al., } \\
\text { 2014) }\end{array}$ & Breast cancer vs no breast cancer & $1.46(1.05,2.03)$ & OR \\
\hline & Gao CM, et al.(Gao et al., 2013) & Breast cancer vs no breast cancer & $1.47(1.18,1.84)$ & OR \\
\hline
\end{tabular}


DOI:10.31557/APJCP.2020.21.4.867

Epidemiology of Breast Cancer in Asia

Table 2. Continued

\begin{tabular}{|c|c|c|c|c|}
\hline Factor & Studies & Comparison & $\begin{array}{l}\text { Reported risk (odds ratio, relative } \\
\text { risk or hazard ratio }[95 \% \text { Confidence } \\
\text { interval]) }\end{array}$ & $\begin{array}{l}\text { Effect } \\
\text { measure }\end{array}$ \\
\hline Increased BMI & $\begin{array}{l}\text { Wang XL, et al.(Wang et al., } \\
\text { 2013b) } \\
\text { Sangrajrang S, et al.(Sangrajrang } \\
\text { et al., 2013) }\end{array}$ & $\begin{array}{l}\text { Breast cancer vs no breast cancer } \\
\text { Breast cancer vs no breast cancer }\end{array}$ & $\begin{array}{l}1.58(1.14,2.19) \\
>25 \mathrm{mg} / \mathrm{m} 21.33(1.07,1.65)\end{array}$ & OR \\
\hline High perceived stress & $\begin{array}{l}\text { Wang L, et al.(Wang et al., } \\
\text { 2013a) }\end{array}$ & Breast cancer vs no breast cancer & $1.65(1.10,2.47)$ & OR \\
\hline \multicolumn{5}{|c|}{ Dietary Intake } \\
\hline Crucian carp & Gao CM, et al.(Gao et al., 2014) & Breast cancer vs no breast cancer & $6.09(3.04,12.2)$ & OR \\
\hline $\begin{array}{l}\text { Consumption of }>30 \mathrm{~g} \\
\text { fat/day }\end{array}$ & $\begin{array}{l}\text { Balasubramaniam SM, et } \\
\text { al.(Balasubramaniam et al., } \\
\text { 2013) }\end{array}$ & Breast cancer vs no breast cancer & $2.4(1.14,5.45)$ & OR \\
\hline Dietary PUFA & $\begin{array}{l}\text { Kiyabu GY, et al.(Kiyabu et al., } \\
\text { 2015) }\end{array}$ & Breast cancer vs no breast cancer & $2.94(1.26,6.89)$ & HR \\
\hline $\begin{array}{l}\text { Consumption of oils } \\
\text { high in saturated fat }\end{array}$ & $\begin{array}{l}\text { Balasubramaniam SM, et } \\
\text { al.(Balasubramaniam et al., } \\
\text { 2013) } \\
\text { Sangrajrang S, et al.(Sangrajrang } \\
\text { et al., 2013) }\end{array}$ & $\begin{array}{l}\text { Breast cancer vs no breast cancer } \\
\text { Breast cancer vs no breast cancer }\end{array}$ & $\begin{array}{l}2.0(1.03,4.52) \\
\text { Not reported }\end{array}$ & OR \\
\hline Dietary cadmium & $\begin{array}{l}\text { Itoh H, et al.(Itoh et al., 2014) } \\
\text { Nagata C, et al.(Nagata et al., } \\
2013 \text { ) }\end{array}$ & $\begin{array}{l}\text { Breast cancer vs no breast cancer } \\
\text { Breast cancer vs no breast cancer }\end{array}$ & $\begin{array}{l}1.94(1.04,3.63) \\
\text { Per } 1.0 u g / g \text { increase in urinary cad- } \\
\text { mium level } 1.67(1.39,2.01)\end{array}$ & OR \\
\hline Sugar intake & $\begin{array}{l}\text { Sulaiman S, et al.(Sulaiman et } \\
\text { al., 2014) }\end{array}$ & Breast cancer vs no breast cancer & $\begin{array}{l}\text { Premenopausal } 1.93(1.53,2.61) \\
\text { Postmenopausal } 1.87(1.03,2.61)\end{array}$ & OR \\
\hline Meat Intake & Ko KP, et al.(Ko et al., 2013) & $\begin{array}{l}\text { BRCA mutation vs no BRCA } \\
\text { mutation }\end{array}$ & BRCA carriers $1.97(1.13,3.44)$ & HR \\
\hline High intake of fried and & $\begin{array}{l}\text { Wang L, et al.(Wang et al., } \\
\text { 2013a) }\end{array}$ & Breast cancer vs no breast cancer & $1.86(1.24,2.77)$ & OR \\
\hline \multicolumn{5}{|c|}{ stir-fried food } \\
\hline Vitamin D deficiency & $\begin{array}{l}\text { Park S, et al.(Park et al., 2015a) } \\
\text { Shi L, et al.(Shi et al., 2014a) }\end{array}$ & $\begin{array}{l}\text { Breast cancer vs no breast cancer } \\
\text { No comparator - retrospective } \\
\text { study }\end{array}$ & $\begin{array}{l}1.27(1.15,1.39) \\
\text { Smoking } 2.78(1.11,6.95) \\
\text { BMI }<231 \mathrm{~kg} / \mathrm{m} 2 \text { (reference) } \\
\text { BMI } 23-<2.5 \mathrm{~kg} / \mathrm{m} 21.12(0.85,1.47) \\
\text { BMI } \geq 27.5 \mathrm{~kg} / \mathrm{m} 21.57(1.02,2.25)\end{array}$ & $\begin{array}{l}\text { OR } \\
\text { OR } \\
\text { OR }\end{array}$ \\
\hline & & PROTECTIVE FACTORS & & \\
\hline Imaging & & & & \\
\hline $\begin{array}{l}\text { Normal breast ultra- } \\
\text { sound }\end{array}$ & Pan L, et al.(Pan et al., 2013) & $\begin{array}{l}\text { Normal breast ultrasound vs abnor- } \\
\text { mal breast ultrasound }\end{array}$ & $0.14(0.09,0.22)$ & OR \\
\hline \multicolumn{5}{|c|}{ Lifestyle and demographic factors } \\
\hline $\begin{array}{l}\text { Higher Healthy Index } \\
\text { score }\end{array}$ & $\begin{array}{l}\text { Shahril MR, et al.(Shahril et al., } \\
\text { 2013) }\end{array}$ & Breast cancer vs no breast cancer & $\begin{array}{l}\text { Premenopausal } 0.34(0.15,0.76) \\
\text { Postmenopausal } 0.20(0.06,0.63)\end{array}$ & OR \\
\hline Underweight BMI & $\begin{array}{l}\text { Sangrajrang S, et al.(Sangrajrang } \\
\text { et al., 2013) }\end{array}$ & Breast cancer vs no breast cancer & $\begin{array}{l}\text { At } 10 \text { years old } 0.70(0.56,0.88) \\
\text { At } 20 \text { years old } 0.74(0.59,0.93)\end{array}$ & OR \\
\hline Regular exercise & $\begin{array}{l}\text { Sangrajrang S, et al.(Sangrajrang } \\
\text { et al., 2013) }\end{array}$ & Breast cancer vs no breast cancer & $0.78(0.68,0.98)$ & OR \\
\hline Literacy & Lan NH, et al.(Lan et al., 2013) & $\begin{array}{l}\text { No comparator - retrospective data } \\
\text { review }\end{array}$ & $0.52(0.89,0.96)$ & HR \\
\hline & & Parity and Ovarian Health & & \\
\hline 1-3 child births & Lee JS, et al.(Lee and Oh, 2014) & $\begin{array}{l}\text { No comparator - retrospective data } \\
\text { review }\end{array}$ & $\begin{array}{l}\text { Luminal A recurrence } 0.56(0.34,0.91) \\
\text { Luminal B recurrence } 0.32(0.17,0.61)\end{array}$ & HR \\
\hline Increasing parity & $\begin{array}{l}\text { Shamsi U, et al.(Shamsi et al., } \\
\text { 2013) }\end{array}$ & Breast cancer vs no breast cancer & $0.90(0.85,0.97)$ & OR \\
\hline $\begin{array}{l}\text { Premature ovarian } \\
\text { failure }\end{array}$ & Wu X, et al.(Wu et al., 2014c) & $\begin{array}{l}\text { Premature ovarian failure vs no } \\
\text { premature ovarian failure }\end{array}$ & $0.59(0.38,0.91)$ & OR \\
\hline \multicolumn{5}{|c|}{ Drug treatments or supplementation } \\
\hline Hormone therapy & Lan NH, et al.(Lan et al., 2013) & $\begin{array}{l}\text { No comparator - retrospective data } \\
\text { review }\end{array}$ & $0.22(0.12,0.41)$ & HR \\
\hline $\begin{array}{l}\text { Vitamin D supplemen- } \\
\text { tation }\end{array}$ & $\begin{array}{l}\text { Shamsi U, et al.(Shamsi et al., } \\
\text { 2013) }\end{array}$ & Breast cancer vs no breast cancer & $\begin{array}{l}<3 \text { years } 0.30(0.12,0.81) \\
>3 \text { years } 0.27(0.13,0.56)\end{array}$ & OR \\
\hline $\begin{array}{l}\text { Use of calcitonin nasal } \\
\text { spray for osteoporosis }\end{array}$ & Sun LM, et al.(Sun et al., 2014d) & $\begin{array}{l}\text { Osteoporosis and cancer vs osteo- } \\
\text { porosis and no cancer }\end{array}$ & $0.35(0.15,0.80)$ & OR \\
\hline $\begin{array}{l}\text { EPA (eicosapentaenoic } \\
\text { acid) }\end{array}$ & $\begin{array}{l}\text { Kiyabu GY, et al.(Kiyabu et al., } \\
\text { 2015) }\end{array}$ & Breast cancer vs no breast cancer & $0.47(0.25,0.89)$ & HR \\
\hline
\end{tabular}


Table 2. Continued

\begin{tabular}{|c|c|c|c|c|}
\hline Factor & Studies & Comparison & $\begin{array}{l}\text { Reported risk (odds ratio, relative } \\
\text { risk or hazard ratio [ } 95 \% \text { Confidence } \\
\text { interval]) }\end{array}$ & $\begin{array}{l}\text { Effect } \\
\text { measure }\end{array}$ \\
\hline \multicolumn{5}{|l|}{ Dietary Intake } \\
\hline Dietary fiber & $\begin{array}{l}\text { Sulaiman et.al. (Sulaiman et al., } \\
\text { 2014) }\end{array}$ & Breast cancer vs no breast cancer & $\begin{array}{l}\text { Premenopausal } 0.31(0.12,0.79) \\
\text { Postmenopausal } 0.23(0.07,0.76)\end{array}$ & OR \\
\hline $\begin{array}{l}\text { Freshwater fish (black } \\
\text { and silver carp) }\end{array}$ & Gao et.al. (Gao et al., 2014) & Breast cancer vs no breast cancer & $\begin{array}{l}\text { Black carp } 0.54(0.33,0.92) \\
\text { Silver carp } 0.19(0.11,0.33)\end{array}$ & OR \\
\hline Choline \& betaine & Zhang et.al. (Zhang et al., 2013a) & Breast cancer vs no breast cancer & $\begin{array}{l}\text { Choline } 0.40(0.28,0.57) \\
\text { Betaine } 0.58(0.42,0.80) \\
\text { Combined choline \& betaine } 0.38 \\
(0.27,0.53)\end{array}$ & OR \\
\hline \multirow[t]{3}{*}{$\begin{array}{l}\text { Soy products \& iso- } \\
\text { flavone }\end{array}$} & Ko et. al.(Ko et al., 2013) & $\begin{array}{l}\text { BRCA mutation vs no BRCA } \\
\text { mutation }\end{array}$ & Soy in BRCA carriers $0.39(0.19,0.79)$ & HR \\
\hline & Li et. al. (Li et al., 2013a) & Breast cancer vs no breast cancer & Isoflavone Patients $0.53(0.33,0.85)$ & OR \\
\hline & Wada K, et al.(Wada et al., 2013) & Breast cancer vs no breast cancer & $\begin{array}{l}\text { Isoflavone Population Controls } 0.43 \\
(0.26,0.71)\end{array}$ & OR \\
\hline Carotenoids & Wang et al. (2014a) & Breast cancer vs no breast cancer & $\begin{array}{l}\text { a-carotene } 0.61(0.43,0.88) \\
\text { b-carotene } 0.54(0.38,0.78) \\
\text { b-cryptoxanthin } 0.38(0.26,0.52) \\
\text { lutein / zeaxanthin } 0.49(0.34,0.71)\end{array}$ & OR \\
\hline
\end{tabular}

alleles) (Chen et al., 2014a), mitochondrial DNA content (Jiang et al., 2014), mitochondrial D-loop insertions (Tipirisetti et al., 2014), and telomere length (Qu et al., 2013). The studies included in this review identified several associations between $\mathrm{BC}$ risk and genes that encode apoptotic regulators (e.g. BIM) (Lin et al., 2015), regulators of DNA stability or repair (e.g. BRCA1, ERCC1, TP53) (Chen et al., 2013a; Liu et al., 2013b; Pei et al., 2014; Sun et al., 2014a; Fu et al., 2015; Haryono et al., 2015; Wu et al., 2015), transcription factors (e.g. CDX2, TBX21) (Tulsyan et al., 2014; Yu et al., 2014; Iqbal et al., 2015), estrogen-related enzymes or receptors (e.g. CYP1A2, CYP19A1, ESR1) (Son et al., 2015; Sun et al., 2015b) and cell surface glycoproteins, growth factors or their receptors (e.g. IGKC, CD44, VEGF, IGF1R, HGF, FGFR2, ESR1) (Choi et al., 2014; Pandey et al., 2014; Siddiqui et al., 2014; Wang et al., 2014c; Yu et al., 2014; Son et al., 2015). Associations were also found for cytokines or regulators of cytokine signaling (e.g. SOCS4, IL6) (Choi et al., 2014; Joshi et al., 2014a), proteins involved in regulating circadian rhythm (e.g. PER3) (Wirth et al., 2014), a ligand-gated ion channel (e.g. CHRNA9) (Hsieh et al., 2014), or transcription factors involved in embryonic stem cell proliferation, renewal, and pluripotency (e.g. NANOG, OCT4) (Tulsyan et al., 2014).

\section{Other risk factors for breast cancer in Asia}

Increased BC risk was associated with insulin use for 3 or more years (mortality risk) among participants from Thailand (Tseng, 2015); polycystic ovary syndrome (Shen et al., 2015); tonsillectomy (Sun et al., 2015a); sleep apnea (Chang et al., 2014); multiple sclerosis among participants from Taiwan (Sun et al., 2014c); inflammatory bowel disease among participants from Asia (Tsai et al., 2015); women who had an organ transplant (Pan et al., 2013); and diabetes among participants from China (Wang et al., 2013b). In addition, a study conducted in China found that hypertension was observed in a higher proportion of $\mathrm{BC}$ patients with type 2 diabetes compared with non-diabetic participants with BC (41.5 versus $26.1 \%, p=0.001$ ) (Wang et al., 2014b) demonstrating a link between breast cancer and other comorbidities. Another study conducted in China showed that premature ovarian failure was associated with decreased incidence of BC among postmenopausal women (Wu et al., 2014c). Higher levels of education have been linked to a reduction in breast cancer risk in Asia. (Lan et al., 2013)

Other investigated risk factors included hormone therapy (Lan et al., 2013), radiotherapy (Chitapanarux et al., 2013), BC-associated depression and anxiety (Wang et al., 2014a), environmental factors, including dichlorodiphenyltrichloroethane (DDT) exposure (Tang et al., 2014), and ratio of second to fourth finger length (Hong et al., 2014).

\section{Discussion}

\section{Non-modifiable risk factors}

The key risk factors for $\mathrm{BC}$ are non-modifiable, the strongest of which is being female (American Cancer Society, 2016). Similarly to the studies in Western population (American Cancer Society, 2016), advancing age has also been identified as a non-modifiable risk factor for developing BC in participants from Asia (Chen et al., 2014b; Kotepui et al., 2014; Virani et al., 2014). The studies identified two periods in which an increased $\mathrm{BC}$ risk emerged; first period at approximately 40-60 years, and a second peak at 85 years and over (Kotepui and Chupeerach, 2013; Chen et al., 2014b; Kotepui et al., 2014; Krishnatreya et al., 2014; Virani et al., 2014; Wu et al., 2014b; Varughese et al., 2015), which may be an important consideration for health service planning.

Approximately $5-10 \%$ of $\mathrm{BC}$ cases are thought to arise from gene mutations; the most common cause of hereditary $\mathrm{BC}$ is an inherited mutation in the $B R C A 1$ and $B R C A 2$ genes (American Cancer Society, 2016). Although the lifetime risk of $\mathrm{BC}$ among those with 
$B R C A 1$ mutations can be as high as $80 \%$, individuals with mutations in $B R C A 1$ or $B R C A 2$ generally have a $55-65 \%$ or $45 \%$ increased risk of $\mathrm{BC}$, respectively (National Cancer Institute, 2017). In 2016, the Asian BRCA consortium released its first paper on the management of hereditary breast and ovarian cancer (HBOC) in 14 Asian nations. This paper highlighted the disparity in the management of HBOC screening (only approximately 4,000 cases of BC in Asia had benefited from genetic services) between nations and recommended that policy makers, healthcare sectors and researchers address the limitations in HBOC testing and management (Nakamura et al., 2016). Results of the studies included in this review which considered the role of $B R C A$ mutation in $\mathrm{BC}$ risk indicated that women with $B C$ and a $B R C A 1$ mutation had an earlier age of disease onset, higher nuclear grade of BC, and younger age of hospitalization (Chen et al., 2013a; Liu et al., 2013b; Pei et al., 2014; Sun et al., 2014a; Fu et al., 2015; Haryono et al., 2015; Wu et al., 2015) when compared to those without the $B R C A$ mutation. Other gene mutations can also lead to $B C$, for example, $C H E K 2$, PTEN, CGH1, STK11 and PALB2 (American Cancer Society, 2016). However, they are generally viewed as being much less common and most do not increase the risk of $\mathrm{BC}$ to the degree seen with the $B R C A$ genes; other non- $B R C A$ mutations have also been linked to increased $B C$. Interestingly, certain gene variations were associated with decreased BC risk (e.g. RAD52, OCT4, FASL, IGFIR, APE1, BARD1, IL4, IL21) (Kang et al., 2013; Liu et al., 2013a; You et al., 2013; Joshi et al., 2014b; Kang et al., 2014; Tulsyan et al., 2014; Xu et al., 2014; Wu et al., 2015), or both increased and decreased risk, depending on the gene variation investigated (e.g. MTHFR, ESR1, $V E G F, F A S, O G G 1, T G F B 1$ ) (Pooja et al., 2013; Kapahi et al., 2014; Lu et al., 2014; Luo et al., 2014; Wang et al., 2014c; Xu et al., 2014; Yu et al., 2014; Lu et al., 2015; Son et al., 2015).

Many women with $\mathrm{BC}$ do not have a family history of the disease. However, having a first-degree relative with $\mathrm{BC}$ is associated with a two-fold increase in risk of $\mathrm{BC}$, and having two first-degree relatives can increase the risk three-fold (American Cancer Society, 2016). Accordingly, we found that family history of BC was associated with an increased risk in studies conducted among participants from China (Zhou et al., 2013; Chen et al., 2014b; Lee et al., 2014; Pei et al., 2014), Pakistan (Shamsi et al., 2013), Hong Kong (Tse et al., 2015), India (Bhadoria et al., 2013), and Korea (Park et al., 2013).

Compared with other races or ethnic origins, Asian, Hispanic and Native American women may have a decreased risk of developing BC (American Cancer Society, 2016). This was supported by two studies which identified a reduced risk of $\mathrm{BC}$ among women from Asia and Malaysia compared with populations of women in the US (Horne et al., 2015; Sung et al., 2015).

Early menarche (before age 12 years) and late menopause (after age 55 years) are associated with increased risk of $\mathrm{BC}$, likely due to a longer lifetime exposure to estrogen and progesterone (American Cancer Society, 2016). Several studies demonstrated a link between menopausal status and $\mathrm{BC}$ risk and was seen across participants from Thailand (Anothaisintawee et al., 2014), India (Chattopadhyay et al., 2014), Pakistan (Song et al., 2014a), and Korea (Park et al., 2013). As expected, the studies showed an increased BC risk in women who had a lower age of menarche (Bhadoria et al., 2013; Chung et al., 2013) and a later age of menopause (Bhadoria et al., 2013; Shamsi et al., 2013; Lee et al., 2014).

An increased risk of $\mathrm{BC}$ was identified in participants with pre-existing medical conditions including multiple sclerosis and polycystic ovary syndrome (Sun et al., 2014c; Shen et al., 2015).

\section{Modifiable risk factors}

Being overweight or obese is associated with an increased risk of $\mathrm{BC}$, especially in women who have reached menopause. This is due to body fat, rather than the ovaries, becoming the primary source of estrogen in menopause (American Cancer Society, 2016). Internationally, several epidemiological studies have found that postmenopausal women with an increased BMI are at an increased BC risk of between 10-60\% (Tahergorabi et al., 2016). In addition, being overweight or obese can increase insulin levels, and higher insulin levels have also been associated with increased risk of BC (American Cancer Society, 2016). Several of the studies in this review showed a correlation between increased BC risk and a high BMI, being overweight or obese, and low level of physical activity (Mizoo et al., 2013; Sangrajrang et al., 2013; Suzuki et al., 2013; Wang et al., 2013a; Wang et al., 2013b; Anothaisintawee et al., 2014; Wada et al., 2014; Fu et al., 2015). In addition one study showed that underweight BMI at a young age (at ages 10 and 20 years) were associated with a decreased risk of BC (Sangrajrang et al., 2013).

In parallel with these observations, physical activity (walking) was associated with decreased risk of BC (Sangrajrang et al., 2013), although the exact amount of exercise needed to induce a protective effect remains unclear (American Cancer Society, 2016).

The connection between $\mathrm{BC}$, diabetes and obesity, was considered in a number of the identified studies. Breast cancer mortality risk was increased in women who had used insulin for more than 3 years (Tseng, 2015); additionally $\mathrm{BC}$ risk was associated with the presence of diabetes (Wang et al., 2013b). when compared to nontamoxifen users (Sun et al., 2014b). Hypertension was observed in a higher proportion of $\mathrm{BC}$ patients with type 2 diabetes when compared to non-diabetic participants with BC (Wang et al., 2014b).

Women who have not had children or who had their first child after age 30 may have a slightly higher BC risk, whereas having many pregnancies and becoming pregnant at an early age may reduce the risk of BC (American Cancer Society, 2016). This was supported by some of the identified studies, in which BC risk increased when first full-term pregnancy was at an age greater than 25 years. Some studies also found that a later age at first live birth, first pregnancy and last pregnancy/birth also increased BC risk (Bhadoria et al., 2013; Huang et al., 2014; Pei et al., 2014). BC risk was found to decrease in women with history of full-term pregnancy (Pei et al., 2014). However, 
one study noted that increased parity and early age at first birth were associated with increased risk of $\mathrm{BC}$ recurrence (Lee and Oh, 2014).

Diet and the use of vitamins are modifiable risk factors with an unclear effect on BC. High consumption of fruits and vegetables was associated with decreased $\mathrm{BC}$ risk (Sangrajrang et al., 2013), and high intake of fatty foods was associated with increased BC risk (Balasubramaniam et al., 2013; Wang et al., 2013a). Data suggested that an intake of vitamin D, dietary fiber and intake of plant- and soy-based products may be protective for BC (Ko et al., 2013; Li et al., 2013a; Wada et al., 2013; Shi et al., 2014a; Sulaiman et al., 2014; Wang et al., 2014a; Park et al., 2015a), whereas high intake of dietary salt, sugar and meat may increase the risk of BC (Ko et al., 2013; Park et al., 2014b; Sulaiman et al., 2014).

There is increasing evidence which supports the link between BC risk and heavy smoking over a long period of time (American Cancer Society, 2016). This was confirmed in several articles demonstrating a clear association between $\mathrm{BC}$ risk and exposure, either active or passive, to tobacco smoke (Mizoo et al., 2013; Pimhanam et al., 2014; Tong et al., 2014; Wada et al., 2015).

\section{Limitations}

There are several limitations to this study. Firstly, due to the large number of articles identified, the results included are based only on the content of article abstracts, as assessed by a single reviewer. Therefore, the results analysis is limited due to some missing data on comparators, effect sizes and other statistical analyses. The missing data also make it difficult to prioritize results according to the strength or quality of included studies and/or data. The strength of the article may also be limited due to the absence of methods to assess risk of bias within individual studies and across studies, and the absence of additional analyses of the extracted data.

In conclusions, this literature review has underscored the importance and relevance of known non-modifiable and modifiable risk factors for Asian women with BC. Furthermore, it has highlighted the differences in epidemiology of $\mathrm{BC}$ in Asian nations when compared to other regions. Only a few studies directly compared BC risk between Asian and non-Asian populations; in the ones that did, there was a decreased risk of $\mathrm{BC}$ among women from Asia compared with women from other countries such as the US.

\section{Acknowledgements}

Editorial services provided by Elizabeth Hutchings RN MNSc from WriteSource Medical writers were funded by Takeda in accordance with Good Publication Practice (GPP3) guidelines (http://ismpp.org/gpp3).

\section{Funding}

Editorial support for the development of this article was provided by Nucleus Global; this support was funded by Takeda Pharmaceutical Company Ltd.
Conflict of interest declaration

HJY, BJC and WH have no conflicts of interest to declare.

\section{References}

Anothaisintawee T, Teerawattananon Y, Wiratkapun C, et al (2014). Development and validation of a breast cancer risk prediction model for Thai women: a cross-sectional study. Asian Pac J Cancer Prev, 15, 6811-7.

Asthana S, Chauhan S, Labani S (2014). Breast and cervical cancer risk in India: an update. Indian J Public Health, 58, 5-10.

Balasubramaniam SM, Rotti SB, Vivekanandam S (2013). Risk factors of female breast carcinoma: a case control study at Puducherry. Indian J Cancer, 50, 65-70.

Bhadoria AS, Kapil U, Sareen N, et al (2013). Reproductive factors and breast cancer: a case-control study in tertiary care hospital of North India. Indian J Cancer, 50, 316-21.

Chang WP, Liu ME, Chang WC, et al (2014). Sleep apnea and the subsequent risk of breast cancer in women: a nationwide population-based cohort study. Sleep Med, 15, 1016-20.

Chattopadhyay S, Siddiqui S, Akhtar MS, et al (2014). Genetic polymorphisms of ESR1, ESR2, CYP17A1, and CYP19A1 and the risk of breast cancer: a case control study from North India. Tumour Biol, 35, 4517-27.

Chen FM, Ou-Yang F, Yang SF, et al (2013a). P53 codon 72 polymorphism in Taiwanese breast cancer patients. Kaohsiung J Med Sci, 29, 259-64.

Chen HT, Wu YC, Chen ST, et al (2014a). Androgen receptor $\mathrm{CAG}$ repeats, non-random $\mathrm{X}$ chromosome inactivation, and loss of heterozygosity at Xq25 in relation to breast cancer risk. BMC Cancer, 14, 144.

Chen JJ, Wang Y, Xue JY, et al (2014b). A clinicopathological study of early-stage synchronous bilateral breast cancer: a retrospective evaluation and prospective validation of potential risk factors. PLoS One, 9, e95185.

Chen YK, Lin CL, Chang YJ, et al (2013b). Cancer risk in patients with Graves' disease: a nationwide cohort study. Thyroid, 23, 879-84.

Chitapanarux I, Tharavichitkul E, Jakrabhandu S, et al (2013). Real-world outcomes of postmastectomy radiotherapy in breast cancer patients with 1-3 positive lymph nodes: a retrospective study. J Radiat Res, 55, 121-8.

Choi J, Song N, Han S, et al (2014). The associations between immunity-related genes and breast cancer prognosis in Korean women. PLoS One, 9, e103593.

Chung S, Park SK, Sung H, et al (2013). Association between chronological change of reproductive factors and breast cancer risk defined by hormone receptor status: results from the Seoul Breast Cancer Study. Breast Cancer Res Treat, 140, 557-65.

Ding X, Jiang M, Jing H, et al (2015). Analysis of serum levels of 15 trace elements in breast cancer patients in Shandong, China Environ Sci Pollut Res Int, 22, 7930-5.

Fei X, Wu J, Kong Z, et al (2015). Urban-rural disparity of breast cancer and socioeconomic risk factors in China. PLoS One, 10, $\mathrm{e} 0117572$.

Fu XJ, Shi XJ, Lin K, et al (2015). Environmental and DNA repair risk factors for breast cancer in South China. Int $J$ Hyg Environ Health, 218, 313-8.

Gao CM, Ding JH, Li SP, et al (2013). Active and passive smoking, and alcohol drinking and breast cancer risk in chinese women. Asian Pac J Cancer Prev, 14, 993-6.

Gao CM, Ding JH, Li SP, et al (2014). Intake of freshwater fish and associated fatty acids and risk of breast cancer. Asian Pac J Cancer Prev, 15, 7879-84. 
Ginsburg O, Bray F, Coleman MP, et al (2017). The global burden of women's cancers: a grand challenge in global health. Lancet, 389, 847-60.

Haryono SJ, Datasena IG, Santosa WB, et al (2015). A pilot genome-wide association study of breast cancer susceptibility loci in Indonesia. Asian Pac J Cancer Prev, 16, 2231-5.

Hassan N, Ho WK, Mariapun S, et al (2015). A cross sectional study on the motivators for Asian women to attend opportunistic mammography screening in a private hospital in Malaysia: the MyMammo study. BMC. Public Health, 15,548 .

Hong L, Zhan-Bing M, Zhi-Yun S, et al (2014). Digit ratio (2D:4D) in Chinese women with breast cancer. Am J Hum Biol, 26, 562-4.

Hori M, Matsuda T, Shibata A, et al (2015). Cancer incidence and incidence rates in Japan in 2009: a study of 32 population-based cancer registries for the Monitoring of Cancer Incidence in Japan (MCIJ) project. Jpn J Clin Oncol, 45, 884-91.

Horne HN, Beena Devi CR, Sung H, et al (2015). Greater absolute risk for all subtypes of breast cancer in the US than Malaysia. Breast Cancer Res Treat, 149, 285-91.

Hsieh YC, Lee CH, Tu SH, et al (2014). CHRNA9 polymorphisms and smoking exposure synergize to increase the risk of breast cancer in Taiwan. Carcinogenesis, 35, 2520-5.

Huang Z, Beeghly-Fadiel A, Gao YT, et al (2014). Associations of reproductive time events and intervals with breast cancer risk: a report from the Shanghai Breast Cancer Study. Int J Cancer, 135, 186-95.

Iqbal M, Khan TA, Maqbool SA (2015). Vitamin D receptor Cdx-2 polymorphism and premenopausal breast cancer risk in southern Pakistani patients. PLoS One, 10, e 0122657.

Itoh H, Iwasaki M, Sawada N, et al (2014). Dietary cadmium intake and breast cancer risk in Japanese women: a case-control study. Int J Hyg Environ Health, 217, 70-7.

Jiang H, Zhao H, Xu H, et al (2014). Peripheral blood mitochondrial DNA content, A10398G polymorphism, and risk of breast cancer in a Han Chinese population. Cancer Sci, 105, 639-45.

Joshi N, Kannan S, Kotian N, et al (2014a). Interleukin 6 $-174 \mathrm{G}>\mathrm{C}$ polymorphism and cancer risk: meta-analysis reveals a site dependent differential influence in Ancestral North Indians. Hum Immunol, 75, 901-8.

Joshi NN, Bhat S, Hake S, et al (2014b). Opposing effects of pro- and anti-inflammatory cytokine gene polymorphisms on the risk for breast cancer in western Indian women: a pilot study. Int J Immunogenet, 41, 242-9.

Jung M, Choi EH, Nam CM, et al (2013). Application of the adjuvant! Online model to Korean breast cancer patients: an assessment of prognostic accuracy and development of an alternative prognostic tool. Ann Surg Oncol, 20, 2615-24.

Kang H, Dai Z, Ma X, et al (2013). A genetic variant in the promoter of APE1 gene $(-656 \mathrm{~T}>\mathrm{G})$ is associated with breast cancer risk and progression in a Chinese population. Gene, 531, 97-100.

Kang HS, Ahn SH, Mishra SK, et al (2014). Association of polymorphisms and haplotypes in the insulin-like growth factor 1 receptor (IGF1R) gene with the risk of breast cancer in Korean women. PLoS One, 9, e84532.

Kapahi R, Guleria K, Sambyal V, et al (2014). Vascular endothelial growth factor (VEGF) gene polymorphisms and breast cancer risk in Punjabi population from North West India. Tumour Biol, 35, 11171-81.

Kasahara Y, Kawai M, Tsuji I, et al (2013). Harms of screening mammography for breast cancer in Japanese women. Breast Cancer, 20, 310-5.
Keramatinia A, Mousavi-Jarrahi SH, Hiteh M, et al (2014). Trends in incidence of breast cancer among women under 40 in Asia. Asian Pac J Cancer Prev, 15, 1387-90.

Kim JH, Choi DH, Park W, et al (2014). Influence of boost radiotherapy in patients with ductal carcinoma in situ breast cancer: a multicenter, retrospective study in Korea (KROG 11-04). Breast Cancer Res Treat, 146, 341-5.

Kim SH, Cho YU, Kim SJ, et al (2013). Low bone density in breast cancer survivors in Korea: prevalence, risk factors and associations with health-related quality of life. Eur $J$ Oncol Nurs, 17, 196-203.

Kiyabu GY, Inoue M, Saito E, et al (2015). Fish, n - 3 polyunsaturated fatty acids and $\mathrm{n}-6$ polyunsaturated fatty acids intake and breast cancer risk: The Japan Public Health Center-based prospective study. Int J Cancer, 137, 2915-26.

Ko KP, Kim SW, Ma SH, et al (2013). Dietary intake and breast cancer among carriers and noncarriers of BRCA mutations in the Korean Hereditary Breast Cancer Study. Am J Clin Nutr, 98, 1493-501.

Kotepui M, Chupeerach C (2013). Age distribution of breast cancer from a Thailand population- based cancer registry. Asian Pac J Cancer Prev, 14, 3815-7.

Kotepui M, Piwkham D, Chupeerach C, et al (2014). Epidemiology and histopathology of benign breast diseases and breast cancer in southern Thailand. Eur J Gynaecol Oncol, 35, 670-5.

Krishnatreya M, Kataki AC, Sharma JD, et al (2014). Descriptive epidemiology of common female cancers in the north East India--a hospital based study. Asian Pac J Cancer Prev, 15, 10735-8.

Kuchiba A, Iwasaki M, Ono H, et al (2014). Global methylation levels in peripheral blood leukocyte DNA by LUMA and breast cancer: a case-control study in Japanese women. $\mathrm{Br}$ J Cancer, 110, 2765-71.

Kwong A, Chau WW, Mang OW, et al (2014). Male breast cancer: a population-based comparison with female breast cancer in Hong Kong, Southern China: 1997-2006. Ann Surg Oncol, 21, 1246-53.

Lan NH, Laohasiriwong W, Stewart JF (2013). Survival probability and prognostic factors for breast cancer patients in Vietnam. Glob Health Action, 6, 1-9.

Lee H, Li JY, Fan JH, et al (2014). Risk factors for breast cancer among Chinese women: a 10-year nationwide multicenter cross-sectional study. J Epidemiol, 24, 67-76.

Lee JS, Oh M (2014). Reproductive factors and subtypes of breast cancer defined by estrogen receptor, progesterone receptor, and human epidermal growth factor receptor 2: a register-based study from Korea. Clin Breast Cancer, 14, 426-34.

Li L, Zhang M, Holman CD (2013a). Population versus hospital controls in the assessment of dietary intake of isoflavone for case-control studies on cancers in China. Nutr Cancer, 65, 390-7.

Li XP, Cao GW, Sun Q, et al (2013b). Cancer incidence and patient survival rates among the residents in the Pudong New Area of Shanghai between 2002 and 2006. Chin J Cancer, 32, 512-9.

Lim VW, Li J, Gong Y, et al (2014). Serum estrogen receptor bioactivity and breast cancer risk among postmenopausal women. Endocr Relat Cancer, 21, 263-73.

Lin CH, Shen CY, Lee JH, et al (2015). High Prevalence of the BIM Deletion Polymorphism in Young Female Breast Cancer in an East Asian Country. PLoS One, 10, e0124908.

Liu H, Zhang H, Sun X, et al (2013a). A cross-sectional study of associations between nonsynonymous mutations of the BARD1 gene and breast cancer in Han Chinese women. Asia Pac J Public Health, 25, 8-14. 
Liu J, Tang X, Li M, et al (2013b). Functional MDM4 rs4245739 genetic variant, alone and in combination with P53 Arg72Pro polymorphism, contributes to breast cancer susceptibility. Breast Cancer Res Treat, 140, 151-7.

$\mathrm{Lu} \mathrm{H}$, Chen D, Hu LP, et al (2014). Estrogen receptor alpha gene polymorphisms and breast cancer risk: a case-control study with meta-analysis combined. Asian Pac J Cancer Prev, 14, 6743-9.

Lu Q, Jiang K, Li Q, et al (2015). Polymorphisms in the MTHFR gene are associated with breast cancer risk and prognosis in a Chinese population. Tumour. Biol, 36, 3757-62.

Luo H, Li Z, Qing Y, et al (2014). Single nucleotide polymorphisms of DNA base-excision repair genes (APE1, OGG1 and XRCC1) associated with breast cancer risk in a Chinese population. Asian Pac J Cancer Prev, 15, 1133-40.

Malvia S, Bagadi SA, Dubey US, et al (2017). Epidemiology of breast cancer in Indian women. Asia Pac J Clin Oncol, 13, 289-95.

Matsuda A, Matsuda T, Shibata A, et al (2014). Cancer incidence and incidence rates in Japan in 2008: a study of 25 population-based cancer registries for the Monitoring of Cancer Incidence in Japan (MCIJ) project. Jpn J Clin Oncol, 44, 388-96.

Mizoo T, Taira N, Nishiyama K, et al (2013). Effects of lifestyle and single nucleotide polymorphisms on breast cancer risk: a case-control study in Japanese women. BMC Cancer, 13, 565.

Nagata C, Nagao Y, Nakamura K, et al (2013). Cadmium exposure and the risk of breast cancer in Japanese women. Breast Cancer Res Treat, 138, 235-9.

Nakamura S, Kwong A, Kim S-W, et al (2016). Current status of the management of hereditary breast and ovarian cancer in Asia: first report by the Asian BRCA consortium. Public Health Genomics, 19, 53-60.

National Cancer Institute (2017). BRCA1 and BRCA2: Cancer Risk and Genetic Testing [Online]. Washington: National Instituted of Health. Available: https://www.cancer.gov/ about-cancer/causes-prevention/genetics/brca-fact-sheet - q2 [Accessed 20 October 2017].

Noh HM, Song YM, Park JH, et al (2013). Metabolic factors and breast cancer risk in Korean women. Cancer Causes Control, 24, 1061-8.

Pan L, Han LL, Tao LX, et al (2013). Clinical risk factor analysis for breast cancer: 568,000 subjects undergoing breast cancer screening in Beijing, 2009. Asian Pac J Cancer Prev, 14, 5325-9.

Pandey JP, Kistner-Griffin E, Black L, et al (2014). IGKC and FcgammaR genotypes and humoral immunity to HER2 in breast cancer. Immunobiology, 219, 113-7.

Park B, Ma SH, Shin A, et al (2013). Korean risk assessment model for breast cancer risk prediction. PLoS One, 8, e76736.

Park B, Shin A, Jung-Choi K, et al (2014a). Correlation of breast cancer incidence with the number of motor vehicles and consumption of gasoline in Korea. Asian Pac J Cancer Prev, 15, 2959-64.

Park JH, Kim YC, Koo HS, et al (2014b). Estimated amount of 24-hour urine sodium excretion is positively correlated with stomach and breast cancer prevalence in Korea. $J$ Korean Med Sci, 29, 131-8.

Park S, Lee DH, Jeon JY, et al (2015a). Serum 25-hydroxyvitamin $\mathrm{D}$ deficiency and increased risk of breast cancer among Korean women: a case-control study. Breast Cancer Res. Treat, 152, 147-54.

Park YH, Lee SJ, Jung HA, et al (2015b). Prevalence and clinical outcomes of young breast cancer (YBC) patients according to intrinsic breast cancer subtypes: Single institutional experience in Korea. Breast J, 24, 213-7.
Pei XH, Yang Z, Lv XQ, et al (2014). Genetic variation in ERCC1 and XPF genes and breast cancer risk. Genet Mol Res, 13, 2259-67.

Pimhanam C, Sangrajrang S, Ekpanyaskul C (2014). Tobacco smoke exposure and breast cancer risk in Thai urban females. Asian Pac J Cancer Prev, 15, 7407-11.

Pooja S, Francis A, Rajender S, et al (2013). Strong impact of TGF-beta1 gene polymorphisms on breast cancer risk in Indian women: a case-control and population-based study. PLoS One, 8, e75979.

Poosari A, Promthet S, Kamsa-ard S, et al (2014). Hormonal contraceptive use and breast cancer in Thai women. J Epidemiol, 24, 216-20.

Qu S, Wen W, Shu XO, et al (2013). Association of leukocyte telomere length with breast cancer risk: nested case-control findings from the Shanghai Women's Health Study. Am J Epidemiol, 177, 617-24.

Sangrajrang S, Chaiwerawattana A, Ploysawang P, et al (2013). Obesity, diet and physical inactivity and risk of breast cancer in Thai women. Asian Pac J Cancer Prev, 14, 7023-7.

Shahril MR, Sulaiman S, Shaharudin SH, et al (2013). Healthy eating index and breast cancer risk among Malaysian women. Eur J Cancer Prev, 22, 342-7.

Shamsi U, Khan S, Usman S, et al (2013). A multicenter matched case control study of breast cancer risk factors among women in Karachi, Pakistan. Asian Pac J Cancer Prev, 14, 183-8.

Shen CC, Yang AC, Hung JH, et al (2015). A nationwide population-based retrospective cohort study of the risk of uterine, ovarian and breast cancer in women with polycystic ovary syndrome. Oncologist, 20, 45-9.

Shi L, Nechuta S, Gao YT, et al (2014a). Correlates of 25-hydroxyvitamin $\mathrm{D}$ among Chinese breast cancer patients. PLoS One, 9, e86467.

Shi XJ, Au WW, Wu KS, et al (2014b). Mortality characteristics and prediction of female breast cancer in China from 1991 to 2011. Asian Pac J Cancer Prev, 15, 2785-91.

Siddiqui S, Chattopadhyay S, Akhtar MS, et al (2014). A study on genetic variants of Fibroblast growth factor receptor 2 (FGFR2) and the risk of breast cancer from North India. PLoS One, 9, e110426.

Singh R, Gupta S, Pawar SB, et al (2014). Evaluation of ER, PR and HER-2 receptor expression in breast cancer patients presenting to a semi urban cancer centre in Western India. J Cancer Res Ther, 10, 26-8.

Sohail A, Kanwal N, Ali M, et al (2013). Effects of glutathioneS-transferase polymorphisms on the risk of breast cancer: a population-based case-control study in Pakistan. Environ. Toxicol. Pharmacol, 35, 143-53.

Son BH, Kim MK, Yun YM, et al (2015). Genetic polymorphism of ESR1 rs2881766 increases breast cancer risk in Korean women. J Cancer Res Clin Oncol, 141, 633-45.

Song N, Choi JY, Sung H, et al (2014a). Heterogeneity of epidemiological factors by breast tumor subtypes in Korean women: a case-case study. Int J Cancer, 135, 669-81.

Song QK, Li J, Huang R, et al (2014b). Age of diagnosis of breast cancer in china: almost 10 years earlier than in the United States and the European union. Asian Pac J Cancer Prev, 15, 10021-5.

Sugawara Y, Kakizaki M, Nagai M, et al (2013). Lactation pattern and the risk for hormone-related female cancer in Japan: the Ohsaki Cohort Study. Eur J Cancer Prev, 22, 187-92.

Sulaiman S, Shahril MR, Wafa SW, et al (2014). Dietary carbohydrate, fiber and sugar and risk of breast cancer according to menopausal status in Malaysia. Asian Pac J Cancer Prev, 15, 5959-64.

Sun L, Liu J, Wang S, et al (2014a). Prevalence of BRCA1 gene 
mutation in breast cancer patients in Guangxi, China. Int $J$ Clin Exp Pathol, 7, 6262-9.

Sun LM, Chen HJ, Li TC, et al (2015a). A nationwide populationbased cohort study on tonsillectomy and subsequent cancer incidence. Laryngoscope, 125, 134-9.

Sun LM, Chen HJ, Liang JA, et al (2014b). Association of tamoxifen use and increased diabetes among Asian women diagnosed with breast cancer. Br J Cancer, 111, 1836-42.

Sun LM, Lin CL, Chung CJ, et al (2014c). Increased breast cancer risk for patients with multiple sclerosis: a nationwide population-based cohort study. Eur J Neurol, 21, 238-44.

Sun LM, Lin MC, Muo CH, et al (2014d). Calcitonin nasal spray and increased cancer risk: a population-based nested case-control study. J Clin Endocrinol Metab, 99, 4259-64.

Sun MY, Du HY, Zhu AN, et al (2015b). Genetic polymorphisms in estrogen-related genes and the risk of breast cancer among Han Chinese women. Int J Mol Sci, 16, 4121-35.

Sung H, Rosenberg PS, Chen WQ, et al (2015). Female breast cancer incidence among Asian and Western populations: more similar than expected. J Natl Cancer Inst, 107, djv107.

Suzuki S, Kojima M, Tokudome S, et al (2013). Obesity/ weight gain and breast cancer risk: findings from the Japan collaborative cohort study for the evaluation of cancer risk. J Epidemiol, 23, 139-45.

Tahergorabi Z, Khazaei M, Moodi M, et al (2016). From obesity to cancer: a review on proposed mechanisms. Cell Biochem Function, 34, 533-45.

Tang M, Zhao M, Shanshan Z, et al (2014). Assessing the underlying breast cancer risk of Chinese females contributed by dietary intake of residual DDT from agricultural soils. Environ Int, 73, 208-15.

Thapa B, Singh Y, Sayami P, et al (2013). Breast cancer in young women from a low risk population in Nepal. Asian Pac $J$ Cancer Prev, 14, 5095-9.

Tipirisetti NR, Govatati S, Pullari P, et al (2014). Mitochondrial control region alterations and breast cancer risk: a study in South Indian population. PLoS One, 9, e85363.

Tong JH, Li Z, Shi J, et al (2014). Passive smoking exposure from partners as a risk factor for $\mathrm{ER}+/ \mathrm{PR}+$ double positive breast cancer in never-smoking Chinese urban women: a hospitalbased matched case control study. PLoS One, 9, e97498.

Tsai MS, Chen HP, Hung CM, et al (2015). Hospitalization for inflammatory bowel disease is associated with increased risk of breast cancer: A nationwide cohort study of an Asian population. Ann Surg Oncol, 22, 1996-2002.

Tse LA, Li M, Chan WC, et al (2015). Familial risks and estrogen receptor-positive breast cancer in Hong Kong Chinese women. PLoS One, 10, e0120741.

Tseng CH (2015). Use of Insulin and mortality from breast cancer among Taiwanese women with diabetes. J Diabetes Res, 2015, 678756.

Tulsyan S, Agarwal G, Lal P, et al (2014). Significant association of combination of OCT4, NANOG, and SOX2 gene polymorphisms in susceptibility and response to treatment in North Indian breast cancer patients. Cancer Chemother Pharmacol, 74, 1065-78.

Varughese AA, Poothiode U, Manjula VD (2015). Descriptive study on selected risk factors and histopathology of breast carcinoma in a tertiary care centre in Kerala, India with special reference to women under 40 years old. Asian Pac $J$ Cancer Prev, 16, 181-4.

Virani S, Sriplung H, Rozek LS, et al (2014). Escalating burden of breast cancer in southern Thailand: analysis of 1990-2010 incidence and prediction of future trends. Cancer Epidemiol, 38, 235-43.

Wada K, Kawachi T, Hori A, et al (2015). Husband's smoking status and breast cancer risk in Japan: From the Takayama study. Cancer Sci, 106, 455-60.

Wada K, Nagata C, Tamakoshi A, et al (2014). Body mass index and breast cancer risk in Japan: a pooled analysis of eight population-based cohort studies. Ann Oncol, 25, 519-24.

Wada K, Nakamura K, Tamai Y, et al (2013). Soy isoflavone intake and breast cancer risk in Japan: from the Takayama study. Int J Cancer, 133, 952-60.

Wang L, Li B, Pan MX, et al (2014a). Specific carotenoid intake is inversely associated with the risk of breast cancer among Chinese women. Br J Nutr, 111, 1686-95.

Wang L, Liao WC, Tsai CJ, et al (2013a). The effects of perceived stress and life style leading to breast cancer. Women Health, 53, 20-40.

Wang RJ, Lu LJ, Jin LB, et al (2014b). Clinicopathologic features of breast cancer patients with type 2 diabetes mellitus in southwest of China. Med Oncol, 31, 788.

Wang XL, Jia CX, Liu LY, et al (2013b). Obesity, diabetes mellitus, and the risk of female breast cancer in Eastern China. World J Surg Oncol, 11, 71.

Wang Y, He Y, Qin Z, et al (2014c). Evaluation of functional genetic variants at $6 \mathrm{q} 25.1$ and risk of breast cancer in a Chinese population. Breast Cancer Res, 16, 422.

Wirth MD, Burch JB, Hebert JR, et al (2014). Case-control study of breast cancer in India: Role of PERIOD3 clock gene length polymorphism and chronotype. Cancer Invest, 32, 321-9.

Wong IO, Schooling CM, Cowling BJ, et al (2015). Breast cancer incidence and mortality in a transitioning Chinese population: current and future trends. $B r J$ Cancer, 112, 167-70.

Wu JQ, Li YY, Ren JC, et al (2014a). Induced abortion and breast cancer: results from a population-based case control study in China. Asian Pac J Cancer Prev, 15, 3635-40.

Wu LZ, Han RQ, Zhou JY, et al (2014b). Incidence and mortality of female breast cancer in Jiangsu, China. Asian Pac. J. Cancer Prev, 15, 2727-32.

Wu X, Cai H, Kallianpur A, et al (2014c). Impact of premature ovarian failure on mortality and morbidity among Chinese women. PLoS One, 9, e89597.

Wu Z, Wang P, Song C, et al (2015). Evaluation of miRNAbinding-site SNPs of MRE11A, NBS1, RAD51 and RAD52 involved in HRR pathway genes and risk of breast cancer in China. Mol Genet Genomics, 290, 1141-53.

Xu Y, Deng Q, He B, et al (2014). The diplotype Fas -1377A/$670 \mathrm{G}$ as a genetic marker to predict a lower risk of breast cancer in Chinese women. Tumour Biol, 35, 9147-61.

Yamashiro H, Takada M, Nakatani E, et al (2014). Prevalence and risk factors of bone metastasis and skeletal related events in patients with primary breast cancer in Japan. Int J Clin Oncol, 19, 852-62.

You Y, Deng J, Zheng J, et al (2013). IL-21 gene polymorphism is associated with the prognosis of breast cancer in Chinese populations. Breast Cancer Res Treat, 137, 893-901.

Yu H, Yang J, Jiao S, et al (2014). T-box transcription factor 21 expression in breast cancer and its relationship with prognosis. Int J Clin Exp Pathol, 7, 6906-13.

Zhang CX, Pan MX, Li B, et al (2013a). Choline and betaine intake is inversely associated with breast cancer risk: a two-stage case-control study in China. Cancer Sci, 104, 250-8.

Zhang X, Li P, Ma W, et al (2013b). Risk factors of recurrence in small-sized, node negative breast cancer in young women: a retrospective study in Chinese population. Sci China Life Sci, 56, 335-40.

Zhou HB, Liu SY, Lei L, et al (2015). Spatio-temporal analysis of female breast cancer incidence in Shenzhen, 2007-2012. Chin J Cancer, 34, 198-204. 
Hyun Jo Youn and Wonshik Han

Zhou W, Pan H, Liang M, et al (2013). Family history and risk of ductal carcinoma in situ and triple negative breast cancer in a Han Chinese population: a case-control study. World $J$ Surg Oncol, 11, 248.

This work is licensed under a Creative Commons AttributionNon Commercial 4.0 International License. 